\title{
Review Article ER Dysfunction and Protein Folding Stress in ALS
}

\author{
Soledad Matus, ${ }^{1}$ Vicente Valenzuela, ${ }^{2,3}$ Danilo B. Medinas, ${ }^{2,3}$ and Claudio Hetz ${ }^{2,3,4}$ \\ ${ }^{1}$ Neurounion Biomedical Foundation, Santiago, Chile \\ ${ }^{2}$ Biomedical Neuroscience Institute, Faculty of Medicine, University of Chile, Santiago, Chile \\ ${ }^{3}$ Center for Molecular Studies of the Cell, Program of Cellular and Molecular Biology, \\ Institute of Biomedical Sciences, University of Chile, Santiago, Chile \\ ${ }^{4}$ Department of Immunology and Infectious Diseases, Harvard School of Public Health, Boston, MA, USA
}

Correspondence should be addressed to Soledad Matus; soledad.matus@neurounion.com and Claudio Hetz; chetz@hsph.harvard.edu

Received 22 May 2013; Accepted 2 September 2013

Academic Editor: Roberto Chiesa

Copyright (c) 2013 Soledad Matus et al. This is an open access article distributed under the Creative Commons Attribution License, which permits unrestricted use, distribution, and reproduction in any medium, provided the original work is properly cited.

Amyotrophic lateral sclerosis (ALS) is the most frequent paralytic disease in adults. Most ALS cases are considered sporadic with no clear genetic component. The disruption of protein homeostasis due to chronic stress responses at the endoplasmic reticulum (ER) and the accumulation of abnormal protein inclusions are extensively described in ALS mouse models and patient-derived tissue. Recent studies using pharmacological and genetic manipulation of the unfolded protein response (UPR), an adaptive reaction against ER stress, have demonstrated a complex involvement of the pathway in experimental models of ALS. In addition, quantitative changes in ER stress-responsive chaperones in body fluids have been proposed as possible biomarkers to monitor the disease progression. Here we review most recent advances attributing a causal role of ER stress in ALS.

\section{Introduction}

Several neurodegenerative disorders, including Alzheimer's disease, Parkinson's disease, Huntington's disease, and amyotrophic lateral sclerosis (ALS), share common features, among them the presence of abnormal protein aggregates and the inclusions containing specific misfolded proteins. The presence of these abnormal protein aggregates has been temporally and spatially correlated with the activation of stress signaling pathway emerging from the endoplasmic reticulum (ER), a cellular reaction named the "unfolded protein response" (UPR). In the last years, ER stress levels and UPR activation in neurodegenerative diseases have been extensively studied. In this review, we focus on recent findings placing ER stress as a key component of neurodegeneration in ALS and discuss the different mechanisms by which the UPR may impact disease progression and the therapeutic potential of manipulating this signaling pathway in ALS.

\section{Amyotrophic Lateral Sclerosis}

ALS is a progressive and deadly adult-onset motoneuron disease characterized by muscle weakness, spasticity, atrophy, paralysis, and premature death $[1,2]$. The pathological hallmark of ALS is the selective degeneration of motoneurons in the spinal ventral horn, most of brainstem nuclei, and cerebral cortex. ALS has an average age of onset around 50 years and estimated incidence of 1-2 cases per 100,000 individuals [1]. ALS is presently incurable with a mean survival time of 1-5 years from diagnosis, often resulting in fatal respiratory dysfunction. The majority of ALS patients lack a defined hereditary genetic component and are considered sporadic (sALS), while approximately $10 \%$ of cases are familial (fALS) [1]. The most common genetic causes of fALS are the recently defined hexanucleotide repeat expansion in the intronic region of C9orf72 and the mutations in the gene encoding cytosolic superoxide dismutase 1 (SOD1), which together 
account for around $50 \%$ of fALS cases. Many other diseasecausative genes have been identified, including TAR DNAbinding protein (TARDBP or TDP-43), fused in sarcoma (FUS/TLS), vesicle-associated membrane protein-associated protein $\mathrm{B}$ (VAPB), among others $[1,3]$. All of these mutations trigger the aggregation of the affected protein, which is associated in part with a gain of neurotoxic activity and possibly neuroinflammatory processes. Overexpression of human fALS-linked SOD1 and TDP-43 mutants in mice recapitulates essential features of the human pathology, provoking age-dependent protein aggregation, paralysis, motoneuron degeneration, and muscle atrophy (reviewed in $[2,4]$ ). Studies in these mouse models of ALS have revealed valuable information about the molecular bases of the disease and, in particular, how the presence of these mutant proteins can trigger ER stress.

Since the same groups of neurons are affected in sALS and fALS leading to a similar pathology, it is predicted that therapies in mutant ALS genetic models may translate to sporadic ALS. In fact, accumulation of misfolded oligomers or protein inclusions containing wild-type (WT) TDP-43, FUS, or SOD1 has been recently shown to be a prominent histopathological feature of sALS (see examples in $[5,6]$ ). Different pathogenic mechanisms have been proposed in ALS including neuroinflammation, glial activation, neuronal trafficking problems, excitotoxicity, mitochondrial dysfunction, and oxidative stress (reviewed in $[2,4]$ ). Interestingly, accumulating evidence from several laboratories points towards a key role of alterations of protein homeostasis in the disease process, in both sALS and fALS (reviewed in [7-9]). In this context, ER stress is emerging as an interesting target for the development of prototypic treatments to ALS. In the next sections, we provide a comprehensive update of the work implicating ER stress to ALS pathogenesis.

\section{ER Stress and UPR Signaling: An Overview}

The ER is the first compartment where secreted and membrane proteins are synthesized and folded. For this process, a large and efficient network of chaperones, foldases, and cofactors are expressed at the ER to promote folding and prevent abnormal aggregation of proteins. The ER also operates as a major intracellular calcium store and plays a crucial role in the synthesis of lipids. A number of stress conditions can interfere with the function of this organelle and cause abnormal oxidative folding at the ER lumen, resulting in a cellular condition termed "ER stress" [10]. ER stress engages the unfolded protein response (UPR), an integrated signal transduction pathway that reestablish homeostasis by increasing the protein folding capacity and quality control mechanisms of the ER [11]. Conversely, chronic ER stress results in apoptosis of irreversibly damaged cells through diverse complementary mechanisms [12].

The UPR is activated by three main stress sensors, including PKR-like ER kinase (PERK), inositol-requiring transmembrane kinase/endonuclease (IRE1), and activating transcription factor 6 (ATF6). IRE1 is an ER located kinase and endoribonuclease conserved from yeast to humans. Upon UPR activation, IRE1 initiates the splicing of the mRNA encoding the transcriptional factor $\mathrm{X}$-Box-binding protein 1 (XBP1), converting it into a potent activator of multiple UPR-responsive genes (termed XBP1s) [13-15]. XBP1s control the expression of genes involved in protein folding, secretion, protein quality control, and ER-associated degradation (ERAD) $[16,17]$. IRE1 $\alpha$ also regulates other signaling events including the downstream activation of JNK, modulating apoptosis and autophagy levels. In addition, IRE1 is able to degrade a subset of mRNA through its RNAse activity on a tissue specific manner (reviewed in [18]).

The activation of the stress sensor PERK reduces protein translation into the ER by phosphorylating eukaryotic initiation factor 2 alpha (eIF2 $\alpha$ ), which in turns contributes to decrease the misfolded protein overload [19]. The phosphorylation of eIF $2 \alpha$ also allows the expression of activating transcription factor 4 (ATF4), a key factor that upregulates a subset of UPR-targeted genes involved in amino acid and redox metabolism, autophagy, protein folding, and apoptosis [20-22] (reviewed in [11, 23]). Among them, CHOP is a key mediator of apoptosis under ER stress [11, 23], which may operate by controlling the expression of several proapoptotic members of the BCL2 family of proteins (i.e., BIM and PUMA) in addition to GADD45 [24]. Sustained PERK signaling also contributes to apoptosis by enhancing oxidative stress and by resuming protein synthesis after prolonged ER stress [25-27].

ATF6 is activated at the ER and then translocates to the Golgi apparatus where it is processed, releasing the cytosolic domain that acts as a transcription factor [11]. ATF6 controls a subset of UPR-targeted genes related to protein folding and quality control mechanisms [28, 29]. Overall, UPR signaling responses integrate information about the nature and intensity of the stress stimuli to modulate the expression of a large spectrum of partially overlapping target genes that orchestrate adaptation to stress or trigger cell death programs [12].

\section{ER Stress Signaling in sALS}

The involvement of ER stress in sporadic ALS can be inferred from correlative studies in human postmortem tissue. Many reports have identified the upregulation and activation of the three main UPR signaling branches, in addition to the description of elevated levels of ER chaperones and cell death signals linked to ER stress [30-34] (see examples in Figure 1). Ilieva et al. showed enhanced phosphorylation of eIF $2 \alpha$ and increased levels of the ER foldase PDIA1 along with elevated levels of oxidized proteins in spinal cord of sporadic ALS patients [32]. We also reported the upregulation of the ER foldase ERp57 in sALS and fALS, in addition to the expression of XBP1s and ATF4 [31]. Other groups also described the upregulation of CHOP in sALS [30, 33] (Figure $1(\mathrm{~d})$ ). In line with the aforementioned observations, augmented levels of PERK, ATF6, and IRE1 have been found [30] (Figure 1(c)).

Additional support for the importance of ER stress in ALS pathogenesis comes from ultrastructural studies [35, 36]. Oyanagi et al. detected distended and fragmented ER cisternae in the affected cells of the anterior horn of the spinal cord [35]. In a recent study, Sasaki observed an 


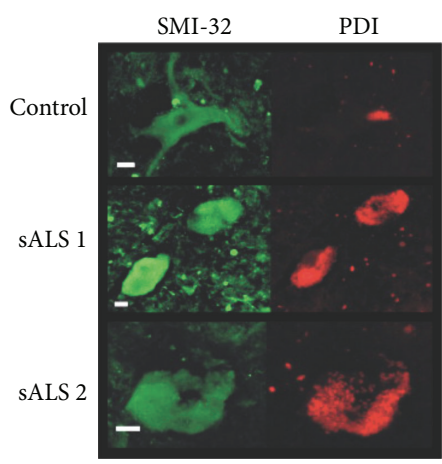

(a)
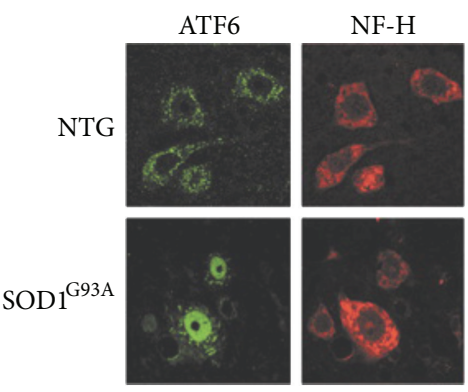
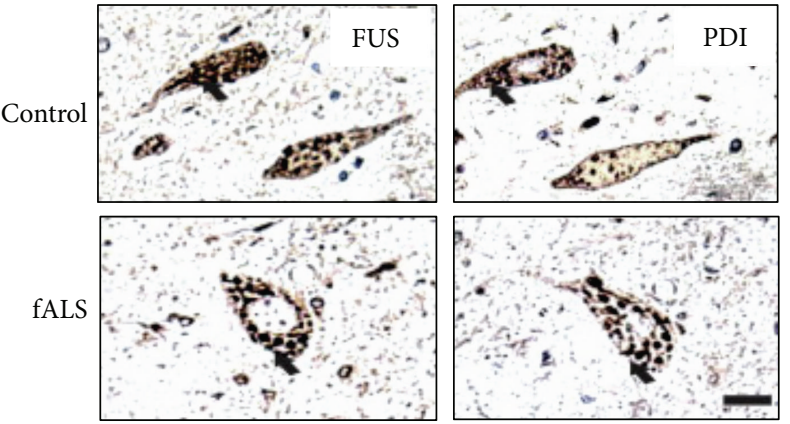

(b)
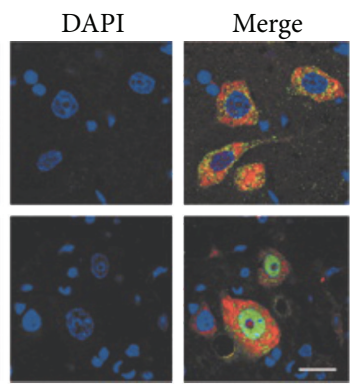

(c)

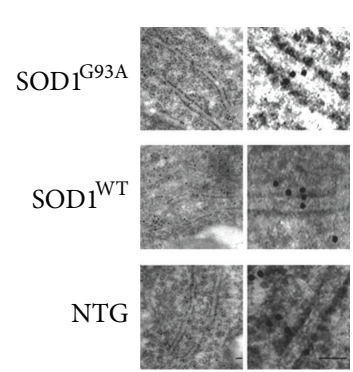

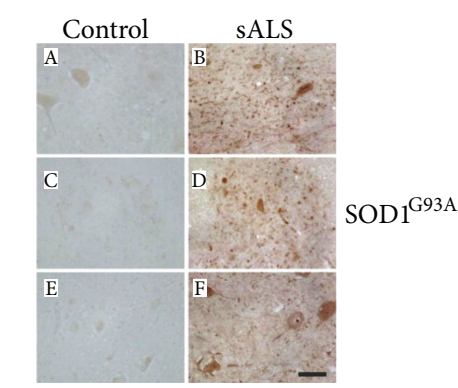

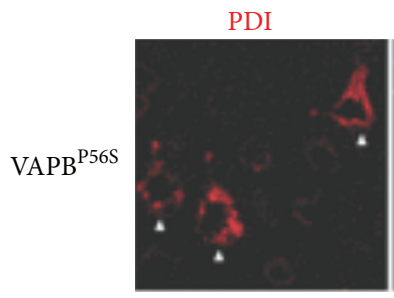

(d)

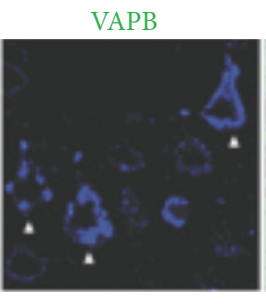

(e)

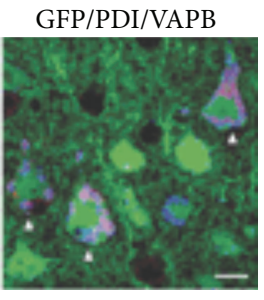

FIGURE 1: UPR activation in ALS human samples and mouse models of the disease. Several examples of published data indicating the activation of the UPR in SALS human samples and animal models. (a) Immunostaining of spinal cord motoneurons with the neurofilament marker SMI-32 showing PDIA1 (PDI) overexpression in samples from two sporadic ALS patients (sALS) compared with healthy subjects. Scale bar, $10 \mu \mathrm{m}$ (from Atkin et al. [30]). (b) Immunohistochemistry of spinal cord section from a familial ALS patient (fALS) with a FUS mutation. The colocalization of FUS protein (left panel) and PDIA1 (PDI) protein (right panel) is indicated with black arrows. Scale bar, $40 \mu \mathrm{m}$ (from Farg et al. [92]). (c) Left panel, immunodetection of the UPR sensor ATF6 (green), neurofilament (NF-H, red), and DAPI (blue) in spinal cord sections from SOD ${ }^{\mathrm{G} 93 \mathrm{~A}}$ mutant mice and nontransgenic control animals (NTG). Scale bar, $20 \mu \mathrm{m}$. Right panel, SOD1 protein detection in ER lumen by immunoelectron microscopy in SOD $1^{\mathrm{G} 93 \mathrm{~A}}$ mutant, SOD1 wild-type (SOD1 ${ }^{\mathrm{WT}}$ ), and nontransgenic (NTG) mice. Scale bar, $50 \mathrm{~nm}$ (from Kikuchi et al. [38]). (d) Left panel, CHOP positive cells detected in spinal cord sections from human sporadic ALS (sALS) patient. Control tissue in (A), (C), and (E). Pictures derived from cervical spinal cord ((A) and (B)), thoracic spinal cord ((C) and (D)), and lumbar spinal cord ((E) and (F)). Scale bars, $65 \mu \mathrm{m}$. In the right panel, immunolocalization of CHOP (green) in anti-ChAT (red) positive spinal cord motoneurons from SOD ${ }^{\mathrm{G} 93 \mathrm{~A}}$ mutant mice. Scale bar: $40 \mu \mathrm{m}$. The areas with a box are shown at higher magnification. Scale bar $10 \mu \mathrm{m}$ (from Ito et al. [33]). (e) Immunostaining of corticospinal motor neurons from 3-month-old VAPB ${ }^{\mathrm{P} 56}$ transgenic mice. Transgene detected with GFP (green), PDIA1 (PDI) (red staining), and VAPB (blue staining). Arrowheads show neurons with accumulation of PDI and VAPB. Scale bar: $20 \mu \mathrm{m}$ (from Aliaga et al. [50]). Copyright authorization was obtained from each journal for all images. 
increased immunostaining for GRP78 (BiP) in affected but also normal-appearing motor neurons from sporadic patients [36]. Strikingly, a detailed examination of ER in normalappearing motor neurons by electron microscopy revealed dilated ER lumen containing amorphous or granular material [36]. Additionally, ribosome-free membranous structures extending from the ER membrane, electron-dense material resembling Bunina bodies, Hirano bodies, and honeycomblike structures were observed in patient samples only [36]. Together, these biochemical and morphological evidence correlate the development of ALS with the markers of ER stress.

\section{UPR Activation in Experimental Models of ALS}

Several laboratories have also shown the occurrence of ER stress in most cellular and animal models of fALS associated with mutations in FUS, TDP-43, SOD1, VAPB, and Ataxin2 (see examples in [37-50]) (Figure 1). Moreover, in addition to ALS, disturbances in the function of the ER are thought to contribute to cell loss in a number of important human diseases including Parkinson's, Huntington's, and Alzheimer's disease [7, 51]. In this section, we discuss mostly in vivo validations of a functional involvement of ER stress in ALS.

In an elegant study from Caroni's group, a systematic transcriptomic analysis was performed using laser dissection of a group of neurons that die early (vulnerable motoneurons) during the course of the disease and a second group that is resistant in a mutant SOD1 model of ALS [52] (Figure 4). This study showed that only affected motoneurons of fALS mouse models were selectively prone to undergo early and chronic ER stress, which was the main molecular signature identified using gene expression profile analysis. Moreover, these changes were detected even before the earliest denervation in asymptomatic animals [52]. In support of this idea, several recent publications suggest that "stressful events" are occurring at the intracellular and intercellular level long before the locomotor defects and the protein aggregation are observed. For example, spinal cord neurons from neonatal SOD1 transgenic mice show hyperexcitability $[53,54]$, which would be one of the earliest abnormalities found so far.

In addition to UPR markers, Saxena et al. also observed that ALS vulnerable neurons specifically engage stressmanagement pathways such as protein ubiquitination and hypoxia-related genes, several weeks before this happens in resistant motoneurons [52]. Furthermore, activation of the UPR in vulnerable motoneurons coincides with the activation of microglia [52] (Figure 4(a)). It is unclear whether resistant motoneurons are protected due to differential disease stress inputs (differential degree of stress) or due to particular cellular mechanisms that generate increased resistance to cellular stress. In conclusion, regardless of the cause of motoneuron stress, it is becoming evident that modulation of protein folding stress or the proteostatic capacity of motoneurons may represent a potent therapeutic target to delay the symptomatic phase of ALS. In this context, the use of gene therapy or small molecules to reinforce the stress response capacity is becoming an interesting tool for disease intervention (Figure 4(c)).

In order to understand the contribution of ER stress and the UPR to ALS, many groups have manipulated UPR components and studied the evolution of the disease (Figure 2). The deficiency of the ER stress-related proapoptotic genes askl, puma, or bim delays ALS in mouse models, possibly by rescuing motoneuron viability $[37,55,56]$. We investigated if deficiency of the transcription factor XBP1 could have an impact on ALS progression by crossbreeding a conditional knockout mouse for XBP1 in the nervous system [57] with transgenic mouse overexpressing mutant SOD1 [31]. Unexpectedly, despite predictions that deletion of this important UPR component would enhance the severity of experimental ALS (i.e., impaired adaptation to ER stress), we observed that the SOD1 mutant offspring that were knockout for XBP1 in the nervous system had delayed disease onset. These effects were associated with reduced accumulation of mutant SOD1 aggregates in vivo and in cell culture models and enhanced autophagy levels [31]. In agreement with this concept, we and others have recently reported that the pharmacological activation of autophagy can improve the survival and disease signs of mouse models of ALS, an effect associated with the clearance of abnormal protein aggregates $[58,59]$. These findings can be contrasted with the unexpected results obtained from the treatment of the mutant SOD1 mice with another autophagy inductor, rapamycin, in which an accelerated progression of the disease was observed [60]. These results may be explained by the fact that the rapamycin target, mTOR (mammalian target of rapamycin), is involved in diverse cellular processes such as regulation of mRNA translation, cell metabolism, and inflammation, among others [61]. Despite these divergent results, autophagy represents an interesting target for future therapeutic development.

Other studies have validated a functional contribution of the UPR to ALS with unexpected results (Figure 2). Remarkably, a treatment of mutant SOD1 transgenic mice with salubrinal [52], a small molecule that enhances eIF2 $\alpha$ phosphorylation [62], led to significant protection against experimental ALS progression [52]. Consistent with this report, perk haploinsufficiency (perk ${ }^{+/-}$mice) exacerbated the severity of experimental ALS, decreasing life span. This phenotype was associated with exacerbated neuronal loss and enhanced mutant SOD1 aggregation [43]. In this study, however, the loss of one perk allele did not decrease the induction of ATF4 at the early symptomatic stage and only partially reduced ATF4 levels at the end stage of the disease [43]. In agreement with this observation the levels of ATF-4 target genes, such chop and bip were not altered in $\mathrm{PERK}^{+/-} / \mathrm{SOD} 1^{\text {mutant }}$ mice [43]. These studies suggest that the effects attributed to perk haploinsufficiency in ALS pathogenesis are mostly related to the inhibition of protein translation through eIF $2 \alpha$ phosphorylation and not due to ATF4 induction.

We also have recently reported the impact of targeting the transcription factor ATF4 in ALS in vivo using a full knockout model. Unexpectedly, ATF4 deficiency reduced the probability of the birth of mutant SOD1 mice, suggesting 


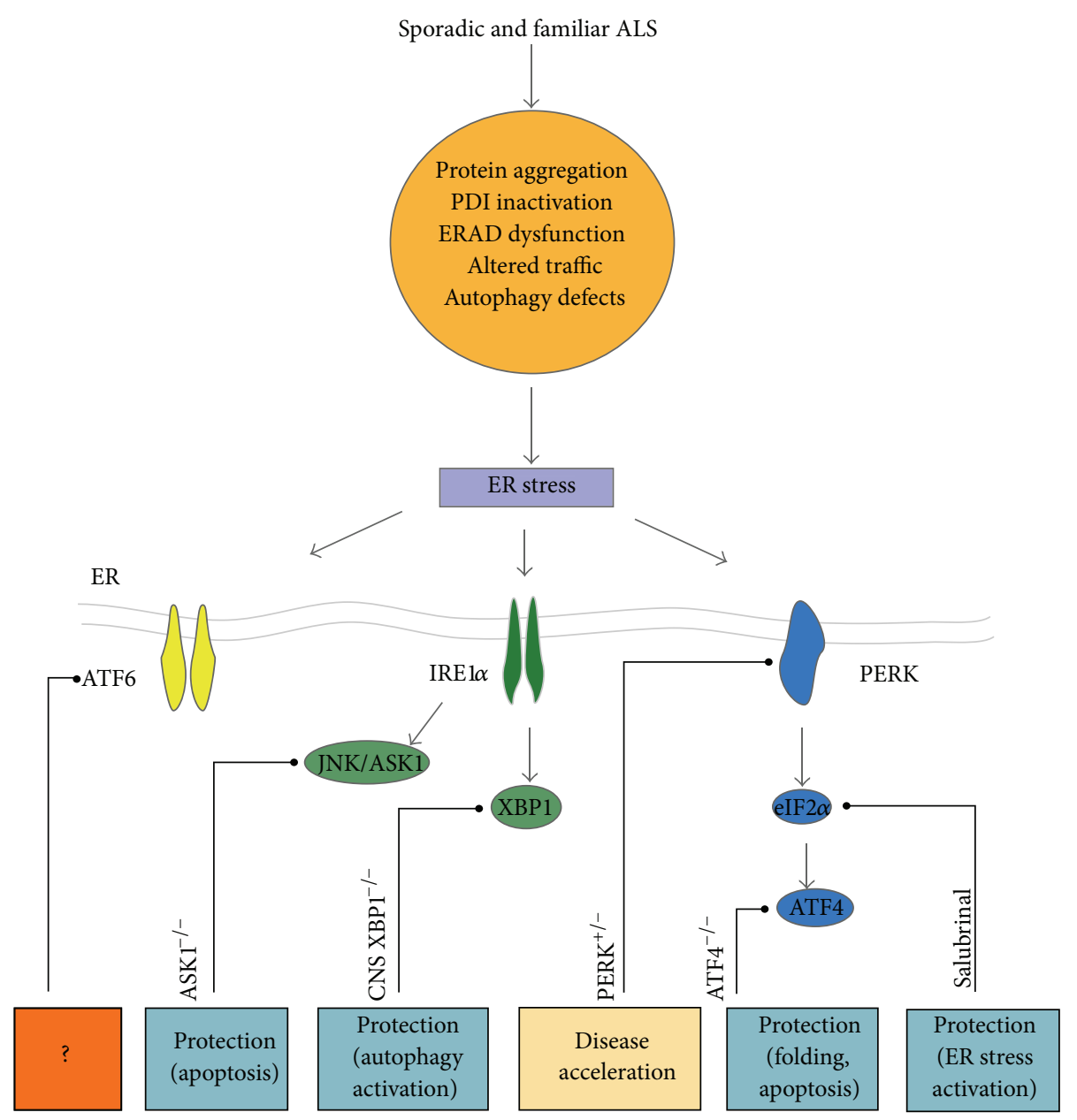

FIgURE 2: Targeting the UPR in ALS. Different factors may induce ER dysfunction in ALS. For example, abnormal protein aggregation/misfolding, PDIs inactivation by nitrosylation, ERAD dysfunction, altered vesicle traffic, and/or autophagy defects represent conditions that could induce ER stress and lead to an adaptive stress response known as the unfolded protein response (UPR) at early disease stages. The manipulation of different UPR components has revealed a functional contribution of distinct ER stress signaling events in preclinical models of ALS. Genetic targeting of ASK1 ( $\mathrm{ASKl}^{-/-}$), a downstream signaling component of IRE1 $\alpha$, protects against the development of experimental ALS decreasing motor neuron death in the spinal cord of mutant SOD $1^{\mathrm{G} 93 \mathrm{~A}}$ mice [56]. The deletion in the CNS of the transcription factor $\mathrm{XBP1}\left(\mathrm{CNS} \mathrm{XBP1}^{-/-}\right)$increases the survival of the mutant SOD1 ${ }^{\mathrm{G} 86 \mathrm{R}}$ mice, associated with reduced accumulation of mutant SOD1 aggregates in vivo and enhanced autophagy levels [31]. PERK haploinsufficiency $\left(\mathrm{PERK}^{+-}\right)$enhanced the severity of experimental ALS, associated with elevated levels of neuronal loss and mutant SOD1 aggregation [43]. The deletion of the transcription factor ATF4 (ATF4 ${ }^{-/-}$) in the SOD1 ${ }^{\mathrm{G} 86 \mathrm{R}}$ mutant mice delays the appearance of the symptoms and the extended animal survival. These effects were associated to changes in the ER protein folding network and apoptotic genes [63]. In a pharmacological strategy, the treatment of mutant SOD1 mice with a small molecule that selectively induces eIF $2 \alpha$ phosphorylation, salubrinal, protects against disease progression [52]. No manipulation of ATF6 in animal models of ALS has been described.

that the UPR may even contribute to mitigating pathological stress during development in this model [63]. On the other hand, the ATF4 knockout/mutant SOD1 transgenic mice that were born showed delayed disease onset and prolonged life span [63]. Consistent with the role of ATF4 in apoptosis, its deficiency completely ablated the induction of BIM and $\mathrm{CHOP}$ in mutant SOD1 mice, in addition to induced quantitative changes in the protein homeostasis network. Conversely, ATF4 deficiency enhanced mutant SOD1 misfolding at the end stage of the disease. Thus, PERK signaling may have differential and contrasting effects on ALS pathogenesis, in which eIF $2 \alpha$ phosphorylation affords protection whereas ATF4 induction may trigger motoneuron apoptosis.

Although the activation of UPR has not been entirely described in animal models expressing TDP-43 mutant proteins [64], in a recent study, the use of drugs to alleviate ER stress showed significant protection against the neurotoxicity induced by mutant TDP- 43 in worm and zebrafish models of ALS [65]. The treatment of these animal models with salubrinal or guanabenz, two drugs that sustain eIF2 $\alpha$ phosphorylation by different mechanisms $[62,66]$, reduced toxicity and improved motility of worms and fishes expressing mutant 
TDP-43 [65]. These results, together with those obtained from pharmacological intervention of eIF $2 \alpha$ in mutant SOD1 mouse models, support the idea that ER stress is a main event in ALS. In summary, these studies illustrate the complex nature of UPR signaling in ALS, clearly demonstrating that targeting specific components of the pathway may have distinct consequences on disease progression [12]. These studies have identified some of the components of the UPR as a potential target to treat ALS.

\section{A Role of the Glia and Oligodendrocyte UPR in ALS?}

The extracellular environment can influence motoneuron fate in the context of ALS as depicted by the interplay between motoneurons and the glia. For example, it is possible to induce ALS pathology in mice overexpressing mutant SOD1 in nonneuronal cells [67]. In cellular assays, supernatant derived from astrocytes/motoneuron cocultures of mutant SOD1 transgenic mice can trigger neuronal death of wild-type neuronal cultures. The toxic factors released from mutant SOD1 primary cells are able to induce hyperexcitability and subsequent cell death [68].

Several studies have shown that the expression of mutant SOD1 in astrocytes or microglia regulates the progression of ALS (see examples in [69-71]). A recent study showed that UPR activation also takes place in these glial cells [72]. ER stress markers can be observed particularly in microglia even at early stages of the disease. These results support the idea that UPR may have a broad impact on noncell autonomous aspects of ALS [72].

Recent reports suggest that oligodendrocytes may also play a relevant role in ALS. Extensive degeneration was reported in the gray matter oligodendrocytes in the spinal cord of mutant SOD1 mice prior to the appearance of disease signs [73]. Similar results were observed in ALS human postmortem tissue [74]. Although new oligodendrocytes were formed, they did not mature and were unable to mediate remyelination. Of note, great advances have been obtained in understanding the role of ER stress in oligodendrocytes in models of multiple sclerosis, where inflammatory reactions trigger demyelination and motoneuron degeneration $[75,76]$. IFN- $\gamma$-dependent activation of the PERK pathway in oligodendrocytes was protective in a mouse model of multiple sclerosis [77]. Moreover, salubrinal also protected against disease progression in the same model [78]. A recent paper confirmed the protective role of PERK pathway against cytotoxic events using a temporally controlled activation of PERK in oligodendrocytes of an experimental model of multiple sclerosis [79]. Similarly, we have recently reported a reduced locomotor recovery in ATF4 or XBP1 knockout models after a spinal cord injury. In addition, gene therapy to deliver active XBP1 into the spinal cord had a significant impact on motor recovery after spinal cord injury which was associated with enhanced oligodendrocyte survival [80]. This is an important finding considering the close relationship of glia and neurons and a possible coordinated/associated stress response between both cell types. These results support the notion that modulating the UPR in a non-cell autonomous manner may also represent an interesting strategy to attenuate ALS progression. This idea remains to be tested.

\section{The PDI Family of Proteins and ALS}

At the early stages of the UPR activation, the folding capacity of the ER is increased through the up-regulation of the ER chaperons such as BiP/Grp78, Grp94, calreticulin (CRT), calnexin (CNX), and several members of the protein disulfide isomerase (PDI) family [81]. These events reduce ER stress levels by enhancing the folding capacity of the ER or by removing terminally misfolded proteins through ERassociated degradation (ERAD) [82]. In the last years, the role of ER resident chaperons and foldases, in particular some members of the PDI family, has gained an important place in the ALS field. Here we discuss most relevant data revealing a participation of these proteins in the ALS.

A recent genetic screening revealed associations of PDIA1 intronic variants as a risk factor to develop ALS [83]. However, no mechanistic studies were provided to determine the possible impact of these genetic alterations on the disease. PDIs are a large protein family comprised of 21 known members of the thioredoxin superfamily, classified based on sequence and structural homology (reviewed in [84]). Most PDIs have a foldase function and catalyzed disulfide bond formation and, as we will discuss later, can also inhibit protein aggregation and modulate cell viability. Of note, several PDI family members have been involved in neurodegenerative disease such as Parkinson's disease, Alzheimer's Disease, prion-related disorders, and Huntington's disease (review in [85]). Importantly, a proteomic analysis of spinal cord tissue of mutant SOD1 mice reporting PDIA1 and ERp57 (also known as Grp58 or PDIA3) as major up-regulated proteins was the first study suggesting a possible participation of PDIs in ALS [86]. These results were later confirmed by independent study [87].

Mutant SOD1 has been shown to accumulate in the ER in vivo $[38,86]$. In addition, the translocation of SOD1 to microsomal fractions has been reconstituted in vitro with purified components [88]. Mutant SOD1 is also secreted to the extracellular space through a classical Golgi-dependent mechanism [41]. Atkin et al. reported a physical interaction between the wild-type and mutant SOD1 and PDIA1 in vivo [86]. They also showed a colocalization of PDIA1 with mutant SOD1 inclusions. This was also observed in spinal cord samples from ALS patients [32, 89]. Similarly, mutant SOD1 was shown to interact with the ER chaperone BiP in the spinal cord of mutant SOD1 transgenic mice [38]. At the functional level, PDIA1 overexpression in cell culture reduced mutant SOD1 aggregation, ER stress, and also induced cell death [90]. In contrast, the inhibition of PDI with the antibiotic bacitracin [91] increased mutant SOD1 inclusions [86], suggesting that PDIA1 prevents the formation of SOD1 aggregates. Similarly, TDP-43 positive inclusions have been shown to colocalize with PDAI1 in sALS samples [89]. ALSlinked FUS mutant has been also shown to induce ER stress, colocalizing with PDIA1 in cell culture and spinal cord tissue from sALS and fALS cases, in addition to animal models of the disease [92]. Moreover, a physical association between 


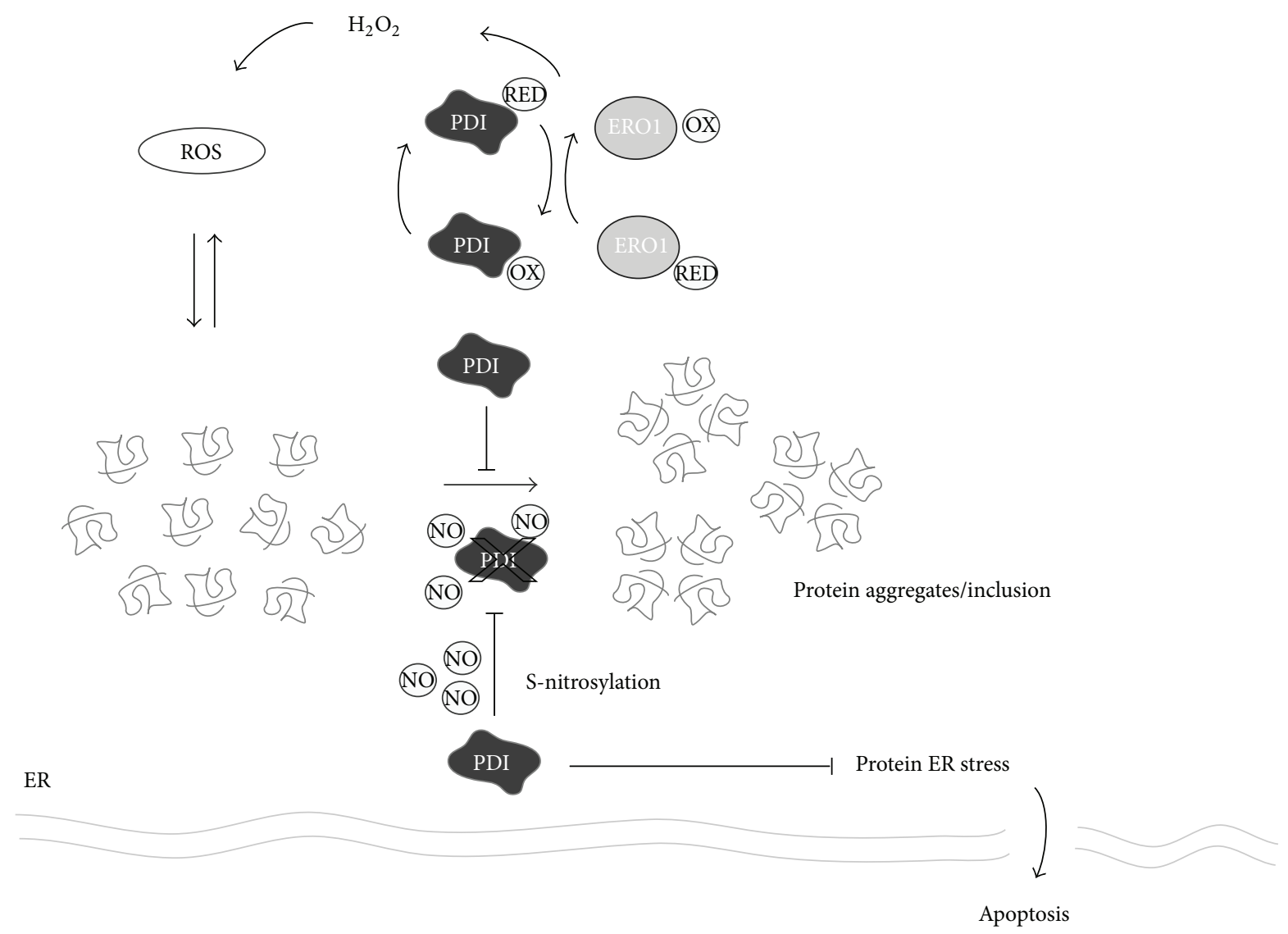

FIGURE 3: Possible role of protein disulfide isomerases (PDIs) in ALS. PDIs are up-regulated in diverse models of ALS in addition to tissue from patients. They also have been suggested as possible biomarkers to monitor disease progression using body fluids. PDIA1, in the figure PDI, colocalized with protein inclusions containing FUS, TDP43 or SOD1 in human tissue and mouse models of ALS. The exact contribution of PDIs to ALS is currently a matter of debate. PDIs could have a protective role through decreasing protein aggregation and global ER stress. S-nitrosylation appears to inactivate PDI and contribute to ER stress. Reactive oxygen species (ROS) produced from redox folding (disulfide bond formation) may also contribute to the generation of protein aggregates.

mutant FUS and PDIA1 was reported [92]. It is still unknown if the manipulation of PDI levels will a_ect the progression of experimental ALS in vivo.

Modification and inactivation of PDIA1 were also reported in spinal cord tissue from sALS and mouse models of the disease [90]. Similar observations were also described before in brain tissue derived from Parkinson's and Alzheimer's disease patients [93]. It was proposed through cell culture studies that PDI nitrosylation may contribute to the disease by inhibiting the protective roles attributed to these foldases. This abnormal modification of PDI could result from altered nitric oxide synthase activity found in mouse models of the disease [94]. Although PDIs are thought to have a neuroprotective activity, one report suggested that PDIA1 and ERp57 may actually have a pro-apoptotic activity in models of Alzheimer and Huntington's disease [95]. Accordingly, UPR activation in microglia correlated with an increase of PDIA1 protein and neurotoxicity [72]. These data suggest that future therapeutic manipulation of the UPR should examine in more detail its impact on glial cells.

The formation of disulfide bonds by PDIs inside the ER requires specific redox conditions and fine balance between the oxidized and reduced states of PDIs [96-100]. The ER is an extremely oxidizing environment compared with the cytoplasm, and the maintenance of its redox state relies on PDI activity of the formation of the disulfide bonds. The generation of disulfide bonds is highly regulated and involves the enzyme ERO1, which is an important oxidase for disulfide formation [101]. The perturbation of the redox status of the ER is deleterious for the proper cell function and there are tight mechanisms to buffer the possible redox fluctuations [102]. We have recently described that ATF4 deficiency alters the redox status of the cell and also the ER as measured by monitoring $\mathrm{H}_{2} \mathrm{O}_{2}$ levels, a subproduct of the PDI/ERO1 cycle [63]. Of note, the treatment of motoneuron cells with the antioxidant trolox is able to revert the enhanced aggregation of mutant SOD1 observed after knocking down ATF4. In addition, overexpression of ERO1 also modulated mutant SOD1 aggregation [63], suggesting that the manipulation of ER redox state can impact the misfolding of mutant SOD1. Taken together, these data suggest that PDIs may play a significant role in ALS by affecting different aspects of cell physiology including protein aggregation, cell survival, and the redox status of the ER (Figure 3). 


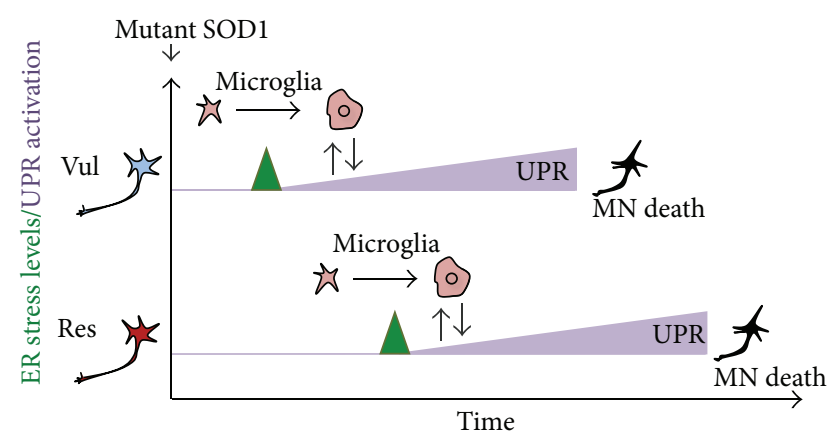

(a)

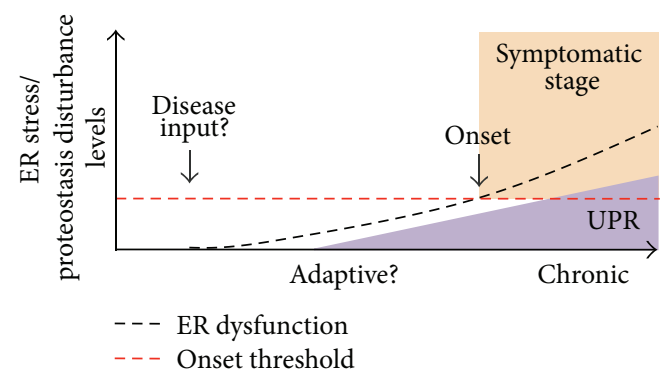

(b)

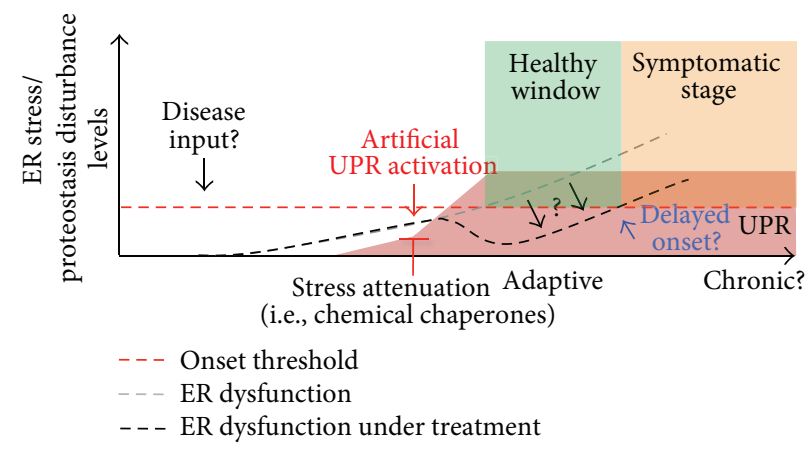

(c)

FIGURE 4: ER stress and the selective neuronal vulnerability in ALS. (a) Schematic representation of ER stress levels (green label) and UPR activation (purple label) in the two subgroups of neurons that have been identified in mutant SOD1 mouse models of ALS: one population that dies early (vul, vulnerable, blue) and another that dies later during disease progression (res, resistant, red). Activation of stress markers is a common feature detected in Vul and Res motoneurons. However, vul motoneurons express these stress markers earlier than de res neurons. The UPR is activated in both subgroups of neurons. UPR activation also correlates with microglial activation in both groups. It is not known what determines the resistance of Res cells in the disease. (b) Time-course of ER stress levels and UPR activation in familial ALS models. ER stress and protein disturbance increase during ALS progression ("ER dysfunction," black dashed line). During the presymptomatic stage of the disease, UPR activation might represent an adaptive response that attenuates ER stress levels. Over time, the stress condition exceeds the capacity of the cell to manage protein folding stress and pro-apoptotic pathways are activated. This shift "onset threshold" in UPR signaling regulation could be associated with motoneuron dysfunction/loss and the onset of the disease. During the symptomatic stage, a strong and chronic UPR activation occurs. (c) Possible therapeutic approaches to modulate the UPR in ALS. An earlystage preventive treatment may modulate UPR levels to enhance the adaptive capacity of motoneurons and reduce ER stress levels or other proteostasis disturbances. This may delay disease onset and disease evolution "healthy window". The therapeutic approaches include gene therapy to deliver active UPR components and the use of smallmolecules that selectively activate specific UPR signaling branches (pharmacologic approaches) or act as chemical chaperones to alleviate global ER stress. This reduction in ER stress levels in motoneurons could also be achieved by modulating glial UPR.

\section{ER Stress Signaling in sALS: Novel Biomarker for Disease Prognosis?}

Early studies have shown that several ER chaperones can be secreted to the extracellular space upon stress [103]. Recently, PDIA1 levels have been reported to be up-regulated in the cerebrospinal fluid (CSF) of ALS patients [30]. Interestingly, Vijayalakshmi et al. showed the induction of ER stress in spinal motor neurons exposed to CSF of sporadic ALS patients [34]. This fact suggests that measuring stress factors in CSF may represent an interesting tool to monitor ALS disease progression. There is a current need for biomarkers of ALS to assess, on a quantitative manner, disease prognosis and the efficacy of clinical trials.

In a recent proteomic screening searching for biomarkers in blood samples from sALS patients, the up-regulation of the ER stress-responsive chaperones PDIA1, ERp57, and other chaperones was observed [104]. Similar changes were also seen in mononuclear cells from blood of mutant SOD1 mice. It was demonstrated that TDP-43, cyclophilin A, and ERp57 are strongly associated with disease course in a longitudinal study in ALS patients and control subjects, ERp57 having the best score [104]. These two studies open the interesting possibility of monitoring stress signatures to diagnose and monitor progression of ALS.

\section{Perspective}

ER dysfunction is currently viewed as a relevant factor driving diverse diseases of the nervous system, representing an important niche for drug discovery. Due to the fact that the type, intensity, and temporality of ER stress stimuli determine 
how the UPR integrates information towards controlling cell fate, this pathway offers interesting targets to modulate both cell survival and death mechanisms. Depending on the disease context, targeting strategies may involve attenuation of ER stress levels, inactivation of pro-apoptotic components of the UPR, or the enhancement of UPR signaling responses toward adaptation to stress (Figure 4). The scenario in ALS is very complex. Genetic and pharmacological manipulation of the pathway in preclinical models of the disease supports the idea that the UPR may contribute to both cell viability of stressed cells and also the elimination of motoneurons when there is irreversible damage. More research is needed to understand the consequence of manipulating the UPR to validate the pathway as a target. For such step, it is essential to define the optimal targets to alleviate ER stress in ALS. Importantly, it is becoming clear that sporadic and familial ALS, regardless of the specific genetic alteration, may converge into alterations on ER function, offering unique therapeutic opportunities. The fact that mutations in PDIA1 gene were recently described in ALS patients suggests a causative role of proteostasis defects at the ER. Supporting this notion, mutations in two important proteins involved in the degradation of misfolded proteins, Ubiquilin1 [105] and p62 [106], have been found in ALS cases. Predicting and defining the possible side effects of manipulating the UPR at the systemic levels remains an important subject for future validation of the pathway as a drug target and move forward into the development of human therapies.

\section{Acknowledgments}

The authors apologize to all colleagues whose work could not be cited owing to space limitations, especially in the introductory parts. This work was funded by FONDECYT 11121524 (Soledad Matus); FONDECYT postdoctoral Grant no. 3130351 (Danilo B. Medinas); The Muscular Dystrophy Association and ALS Therapy Alliance, Millennium Institute no. P09-015-F, FONDECYT no. 1100176, ACT1109; and FONDEF D11I1007 (Claudio Hetz) FONDECYT USA2013003 (Claudio Hetz). They also thank Michael J. Fox Foundation for Parkinson's Research and Alzheimer Disease Association (Claudio Hetz). Vicente Valenzuela received a CONICYT Ph.D. fellowship.

\section{References}

[1] P. M. Andersen and A. Al-Chalabi, "Clinical genetics of amyotrophic lateral sclerosis: what do we really know?" Nature Reviews Neurology, vol. 7, no. 11, pp. 603-615, 2011.

[2] P. Pasinelli and R. H. Brown, "Molecular biology of amyotrophic lateral sclerosis: insights from genetics," Nature Reviews Neuroscience, vol. 7, no. 9, pp. 710-723, 2006.

[3] M. R. Turner, O. Hardiman, M. Benatar et al., "Controversies and priorities in amyotrophic lateral sclerosis," The Lancet Neurology, vol. 12, pp. 310-322, 2013.

[4] S. Boillée, C. Vande Velde, and D. Cleveland, "ALS: a disease of motor neurons and their nonneuronal neighbors," Neuron, vol. 52, no. 1, pp. 39-59, 2006.

[5] D. A. Bosco, G. Morfini, N. M. Karabacak et al., "Wild-type and mutant SOD1 share an aberrant conformation and a common pathogenic pathway in ALS," Nature Neuroscience, vol. 13, no. 11, pp. 1396-1403, 2010.

[6] I. R. A. Mackenzie, R. Rademakers, and M. Neumann, “TDP43 and FUS in amyotrophic lateral sclerosis and frontotemporal dementia," The Lancet Neurology, vol. 9, no. 10, pp. 995-1007, 2010.

[7] S. Matus, L. H. Glimcher, and C. Hetz, "Protein folding stress in neurodegenerative diseases: a glimpse into the ER," Current Opinion in Cell Biology, vol. 23, no. 2, pp. 239-252, 2011.

[8] M. Nassif, S. Matus, K. Castillo, and C. Hetz, "Amyotrophic lateral sclerosis pathogenesis: a journey through the secretory pathway," Antioxidants and Redox Signaling, vol. 13, no. 12, pp. 1955-1989, 2010.

[9] A. K. Walker and J. D. Atkin, "Stress signaling from the endoplasmic reticulum: a central player in the pathogenesis of amyotrophic lateral sclerosis," IUBMB Life, vol. 63, no. 9, pp. 754-763, 2011.

[10] C. Hetz, F. Martinon, D. Rodriguez, and L. H. Glimcher, "The unfolded protein response: integrating stress signals through the stress sensor IRE1 $\alpha$," Physiological Reviews, vol. 91, no. 4, pp. 1219-1243, 2011.

[11] P. Walter and D. Ron, "The unfolded protein response: from stress pathway to homeostatic regulation," Science, vol. 334, no. 6059, pp. 1081-1086, 2011.

[12] C. Hetz, "The unfolded protein response: controlling cell fate decisions under ER stress and beyond," Nature Reviews Molecular Cell Biology, vol. 13, no. 2, pp. 89-102, 2012.

[13] M. Calfon, H. Zeng, F. Urano et al., "IRE1 couples endoplasmic reticulum load to secretory capacity by processing the XBP-1 mRNA," Nature, vol. 415, no. 6867, pp. 92-96, 2002.

[14] K. Lee, W. Tirasophon, X. Shen et al., "IRE1-mediated unconventional mRNA splicing and S2P-mediated ATF6 cleavage merge to regulate XBP1 in signaling the unfolded protein response," Genes and Development, vol. 16, no. 4, pp. 452-466, 2002.

[15] H. Yoshida, T. Matsui, A. Yamamoto, T. Okada, and K. Mori, "XBP1 mRNA is induced by ATF6 and spliced by IRE1 in response to ER stress to produce a highly active transcription factor," Cell, vol. 107, no. 7, pp. 881-891, 2001.

[16] D. Acosta-Alvear, Y. Zhou, A. Blais et al., "XBP1 controls diverse cell type- and condition-specific transcriptional regulatory networks," Molecular Cell, vol. 27, no. 1, pp. 53-66, 2007.

[17] A.-H. Lee, N. N. Iwakoshi, and L. H. Glimcher, "XBP-1 regulates a subset of endoplasmic reticulum resident chaperone genes in the unfolded protein response," Molecular and Cellular Biology, vol. 23, no. 21, pp. 7448-7459, 2003.

[18] D. Ron and P. Walter, "Signal integration in the endoplasmic reticulum unfolded protein response," Nature Reviews Molecular Cell Biology, vol. 8, no. 7, pp. 519-529, 2007.

[19] H. P. Harding, Y. Zhang, and D. Ron, "Protein translation and folding are coupled by an endoplasmic-reticulum-resident kinase," Nature, vol. 397, pp. 271-274, 1999.

[20] H. P. Harding, Y. Zhang, H. Zeng et al., "An integrated stress response regulates amino acid metabolism and resistance to oxidative stress," Molecular Cell, vol. 11, no. 3, pp. 619-633, 2003.

[21] H. Zinszner, M. Kuroda, X. Wang et al., "CHOP is implicated in programmed cell death in response to impaired function of the endoplasmic reticulum," Genes and Development, vol. 12, no. 7, pp. 982-995, 1998.

[22] H. P. Harding, I. Novoa, Y. Zhang et al., "Regulated translation initiation controls stress-induced gene expression in mammalian cells," Molecular Cell, vol. 6, no. 5, pp. 1099-1108, 2000. 
[23] I. Tabas and D. Ron, "Integrating the mechanisms of apoptosis induced by endoplasmic reticulum stress," Nature Cell Biology, vol. 13, no. 3, pp. 184-190, 2011.

[24] H. Urra, E. Dufey, F. Lisbona, D. Rojas-Rivera, and C. Hetz, "When ER stress reaches a dead end," Biochimica et Biophysica Acta. In press.

[25] J. Han, S. H. Back, J. Hur et al., "ER-stress-induced transcriptional regulation increases protein synthesis leading to cell death," Nature Cell Biology, vol. 15, pp. 481-490, 2013.

[26] S. J. Marciniak, C. Y. Yun, S. Oyadomari et al., "CHOP induces death by promoting protein synthesis and oxidation in the stressed endoplasmic reticulum," Genes and Development, vol. 18, no. 24, pp. 3066-3077, 2004.

[27] T. Verfaillie, N. Rubio, A. D. Garg et al., "PERK is required at the ER-mitochondrial contact sites to convey apoptosis after ROSbased ER stress," Cell Death \& Differentiation, vol. 19, no. 11, pp. 1880-1891, 2012.

[28] X. Chen, J. Shen, and R. Prywes, "The luminal domain of ATF6 senses endoplasmic reticulum (ER) stress and causes translocation of ATF6 from the er to the Golgi," The Journal of Biological Chemistry, vol. 277, no. 15, pp. 13045-13052, 2002.

[29] K. Haze, H. Yoshida, H. Yanagi, T. Yura, and K. Mori, "Mammalian transcription factor ATF6 is synthesized as a transmembrane protein and activated by proteolysis in response to endoplasmic reticulum stress," Molecular Biology of the Cell, vol. 10, no. 11, pp. 3787-3799, 1999.

[30] J. D. Atkin, M. A. Farg, A. K. Walker, C. McLean, D. Tomas, and M. K. Horne, "Endoplasmic reticulum stress and induction of the unfolded protein response in human sporadic amyotrophic lateral sclerosis," Neurobiology of Disease, vol. 30, no. 3, pp. 400407, 2008.

[31] C. Hetz, P. Thielen, S. Matus et al., "XBP-1 deficiency in the nervous system protects against amyotrophic lateral sclerosis by increasing autophagy," Genes and Development, vol. 23, no. 19, pp. 2294-2306, 2009.

[32] E. V. Ilieva, V. Ayala, M. Jové et al., "Oxidative and endoplasmic reticulum stress interplay in sporadic amyotrophic lateral sclerosis," Brain, vol. 130, no. 12, pp. 3111-3123, 2007.

[33] Y. Ito, M. Yamada, H. Tanaka et al., "Involvement of CHOP, an ER-stress apoptotic mediator, in both human sporadic ALS and ALS model mice," Neurobiology of Disease, vol. 36, no. 3, pp. 470-476, 2009.

[34] K. Vijayalakshmi, P. A. Alladi, S. Ghosh et al., "Evidence of endoplasmic reticular stress in the spinal motor neurons exposed to CSF from sporadic amyotrophic lateral sclerosis patients," Neurobiology of Disease, vol. 41, no. 3, pp. 695-705, 2011.

[35] K. Oyanagi, M. Yamazaki, H. Takahashi et al., "Spinal anterior horn cells in sporadic amyotrophic lateral sclerosis show ribosomal detachment from, and cisternal distention of the rough endoplasmic reticulum," Neuropathology and Applied Neurobiology, vol. 34, no. 6, pp. 650-658, 2008.

[36] S. Sasaki, "Endoplasmic reticulum stress in motor neurons of the spinal cord in sporadic amyotrophic lateral sclerosis," Journal of Neuropathology and Experimental Neurology, vol. 69, no. 4, pp. 346-355, 2010.

[37] D. Kieran, I. Woods, A. Villunger, A. Strasser, and J. H. M. Prehn, "Deletion of the BH3-only protein puma protects motoneurons from ER stress-induced apoptosis and delays motoneuron loss in ALS mice," Proceedings of the National Academy of Sciences of the United States of America, vol. 104, no. 51, pp. 20606-20611, 2007.
[38] H. Kikuchi, G. Almer, S. Yamashita et al., "Spinal cord endoplasmic reticulum stress associated with a microsomal accumulation of mutant superoxide dismutase-1 in an ALS model," Proceedings of the National Academy of Sciences of the United States of America, vol. 103, no. 15, pp. 6025-6030, 2006.

[39] A. Mori, S. Yamashita, K. Uchino et al., "Derlin-1 overexpression ameliorates mutant SOD1-induced endoplasmic reticulum stress by reducing mutant SOD1 accumulation," Neurochemistry International, vol. 58, no. 3, pp. 344-353, 2011.

[40] T. Nagata, H. Ilieva, T. Murakami et al., "Increased ER stress during motor neuron degeneration in a transgenic mouse model of amyotrophic lateral sclerosis," Neurological Research, vol. 29, no. 8, pp. 767-771, 2007.

[41] M. Urushitani, A. Sik, T. Sakurai, N. Nukina, R. Takahashi, and J.-P. Julien, "Chromogranin-mediated secretion of mutant superoxide dismutase proteins linked to amyotrophic lateral sclerosis," Nature Neuroscience, vol. 9, no. 1, pp. 108-118, 2006.

[42] A. S. Vlug, E. Teuling, E. D. Haasdijk, P. French, C. C. Hoogenraad, and D. Jaarsma, "ATF3 expression precedes death of spinal motoneurons in amyotrophic lateral sclerosis-SOD1 transgenic mice and correlates with c-Jun phosphorylation, CHOP expression, somato-dendritic ubiquitination and Golgi fragmentation," European Journal of Neuroscience, vol. 22, no. 8, pp. 1881-1894, 2005.

[43] L. Wang, B. Popko, and R. P. Roos, "The unfolded protein response in familial amyotrophic lateral sclerosis," Human Molecular Genetics, vol. 20, no. 5, pp. 1008-1015, 2011.

[44] H. Wootz, I. Hansson, L. Korhonen, and D. Lindholm, "XIAP decreases caspase-12 cleavage and calpain activity in spinal cord of ALS transgenic mice," Experimental Cell Research, vol. 312, no. 10, pp. 1890-1898, 2006.

[45] H. Wootz, I. Hansson, L. Korhonen, U. Näpänkangas, and D. Lindholm, "Caspase-12 cleavage and increased oxidative stress during motoneuron degeneration in transgenic mouse model of ALS," Biochemical and Biophysical Research Communications, vol. 322, no. 1, pp. 281-286, 2004.

[46] M. A. Farg, K. Y. Soo, S. T. Warraich et al., "Ataxin-2 interacts with FUS and intermediate-length polyglutamine expansions enhance FUS-related pathology in amyotrophic lateral sclerosis," Human Molecular Genetics, vol. 22, pp. 717-728, 2013.

[47] C. Gkogkas, S. Middleton, A. M. Kremer et al., "VAPB interacts with and modulates the activity of ATF6," Human Molecular Genetics, vol. 17, no. 11, pp. 1517-1526, 2008.

[48] K. Langou, A. Moumen, C. Pellegrino et al., "AAV-mediated expression of wild-type and ALS-linked mutant VAPB selectively triggers death of motoneurons through a Ca2+dependent ER-associated pathway," Journal of Neurochemistry, vol. 114, no. 3, pp. 795-809, 2010.

[49] H. Suzuki, K. Kanekura, T. P. Levine et al., "ALS-linked P56S$\mathrm{VAPB}$, an aggregated loss-of-function mutant of VAPB, predisposes motor neurons to ER stress-related death by inducing aggregation of co-expressed wild-type VAPB," Journal of Neurochemistry, vol. 108, no. 4, pp. 973-985, 2009.

[50] L. Aliaga, C. Lai, J. Yu et al., "Amyotrophic lateral sclerosisrelated VAPB P56S mutation differentially affects the function and survival of corticospinal and spinal motor neurons," Human Molecular Genetics, 2013.

[51] B. D. Roussel, A. J. Kruppa, E. Miranda et al., "Endoplasmic reticulum dysfunction in neurological disease," The Lancet Neurology, vol. 12, pp. 105-118, 2013. 
[52] S. Saxena, E. Cabuy, and P. Caroni, "A role for motoneuron subtype-selective ER stress in disease manifestations of FALS mice," Nature Neuroscience, vol. 12, no. 5, pp. 627-636, 2009.

[53] C. Bories, J. Amendola, B. Lamotte d'Incamps, and J. Durand, "Early electrophysiological abnormalities in lumbar motoneurons in a transgenic mouse model of amyotrophic lateral sclerosis," European Journal of Neuroscience, vol. 25, no. 2, pp. 451-459, 2007.

[54] B. van Zundert, M. H. Peuscher, M. Hynynen et al., "Neonatal neuronal circuitry shows hyperexcitable disturbance in a mouse model of the adult-onset neurodegenerative disease amyotrophic lateral sclerosis," The Journal of Neuroscience, vol. 28, no. 43, pp. 10864-10874, 2008.

[55] C. Hetz, J. Castilla, and C. Soto, "Perturbation of endoplasmic reticulum homeostasis facilitates prion replication," The Journal of Biological Chemistry, vol. 282, no. 17, pp. 12725-12733, 2007.

[56] H. Nishitoh, H. Kadowaki, A. Nagai et al., "ALS-linked mutant SOD1 induces ER stress- and ASK1-dependent motor neuron death by targeting Derlin-1," Genes and Development, vol. 22, no. 11, pp. 1451-1464, 2008.

[57] C. Hetz, A. H. Lee, D. Gonzalez-Romero et al., "Unfolded protein response transcription factor XBP-1 does not influence prion replication or pathogenesis," Proceedings of the National Academy of Sciences of the United States of America, vol. 105, pp. 757-762, 2008.

[58] K. Castillo, M. Nassif, V. Valenzuela et al., “Trehalose delays the progression of amyotrophic lateral sclerosis by enhancing autophagy in motoneurons," Autophagy, vol. 9, no. 9, 2013.

[59] I. F. Wang, B. S. Guo, Y. C. Liu et al., "Autophagy activators rescue and alleviate pathogenesis of a mouse model with proteinopathies of the TAR DNA-binding protein 43," Proceedings of the National Academy of Sciences of the United States of America, vol. 109, pp. 15024-15029, 2012.

[60] X. Zhang, L. Li, S. Chen et al., "Rapamycin treatment augments motor neuron degeneration in SOD1 G93A mouse model of amyotrophic lateral sclerosis," Autophagy, vol. 7, no. 4, pp. 412425, 2011.

[61] M. Laplante and D. M. Sabatini, "MTOR signaling in growth control and disease," Cell, vol. 149, no. 2, pp. 274-293, 2012.

[62] M. Boyce, K. F. Bryant, C. Jousse et al., "A selective inhibitor of elF2 $\alpha$ dephosphorylation protects cells from ER stress," Science, vol. 307, no. 5711, pp. 935-939, 2005.

[63] S. Matus, E. Lopez, V. Valenzuela, M. Nassif, and C. Hetz, "Functional contribution of the transcription factor ATF4 to the pathogenesis of amyotrophic lateral sclerosis," PLoS One, vol. 8, no. 7, Article ID e66672, 2013.

[64] J. Tong, C. Huang, F. Bi et al., "XBP1 depletion precedes ubiquitin aggregation and Golgi fragmentation in TDP-43 transgenic rats," Journal of Neurochemistry, vol. 123, pp. 406-416, 2012.

[65] A. Vaccaro, S. A. Patten, D. Aggad et al., "Pharmacological reduction of ER stress protects against TDP-43 neuronal toxicity in vivo," Neurobiology of Disease, vol. 55, pp. 64-75, 2013.

[66] P. Tsaytler, H. P. Harding, D. Ron, and A. Bertolotti, "Selective inhibition of a regulatory subunit of protein phosphatase 1 restores proteostasis," Science, vol. 332, no. 6025, pp. 91-94, 2011.

[67] A. M. Clement, M. D. Nguyen, E. A. Roberts et al., "Wild-type nonneuronal cells extend survival of SOD1 mutant motor neurons in ALS mice," Science, vol. 302, no. 5642, pp. 113-117, 2003.

[68] E. Fritz, P. Izaurieta, A. Weiss et al., "Mutant SOD1-expressing astrocytes release toxic factors that trigger motor neuron death by inducing hyper-excitability," Journal of Neurophysiology, vol. 109, no. 11, pp. 2803-2814, 2013.
[69] S. Boillée and D. W. Cleveland, "Revisiting oxidative damage in ALS: Microglia, Nox, and mutant SOD1," The Journal of Clinical Investigation, vol. 118, no. 2, pp. 474-478, 2008.

[70] L. Wang, D. H. Gutmann, and R. P. Roos, "Astrocyte loss of mutant SOD1 delays ALS disease onset and progression in G85R transgenic mice," Human Molecular Genetics, vol. 20, no. 2, Article ID ddq463, pp. 286-293, 2011.

[71] K. Yamanaka, S. Boillee, E. A. Roberts et al., "Mutant SOD1 in cell types other than motor neurons and oligodendrocytes accelerates onset of disease in ALS mice," Proceedings of the National Academy of Sciences of the United States of America, vol. 105, no. 21, pp. 7594-7599, 2008.

[72] M. Jaronen, P. Vehvilainen, T. Malm et al., "Protein disulfide isomerase in ALS mouse glia links protein misfolding with NADPH oxidase-catalyzed superoxide production," Human Molecular Genetics, vol. 22, pp. 646-655, 2013.

[73] S. H. Kang, Y. Li, M. Fukaya et al., "Degeneration and impaired regeneration of gray matter oligodendrocytes in amyotrophic lateral sclerosis," Nature Neuroscience, vol. 16, pp. 571-579, 2013.

[74] T. Philips, A. Bento-Abreu, A. Nonneman et al., "Oligodendrocyte dysfunction in the pathogenesis of amyotrophic lateral sclerosis," Brain, vol. 136, pp. 471-482, 2013.

[75] W. Lin, H. P. Harding, D. Ron, and B. Popko, "Endoplasmic reticulum stress modulates the response of myelinating oligodendrocytes to the immune cytokine interferon- $\gamma$," Journal of Cell Biology, vol. 169, no. 4, pp. 603-612, 2005.

[76] J. M. McMahon, S. McQuaid, R. Reynolds, and U. F. FitzGerald, "Increased expression of ER stress- and hypoxia-associated molecules in grey matter lesions in multiple sclerosis," Multiple Sclerosis, vol. 18, pp. 1437-1447, 2012.

[77] W. Lin, S. L. Bailey, H. Ho et al., “The integrated stress response prevents demyelination by protecting oligodendrocytes against immune-mediated damage," The Journal of Clinical Investigation, vol. 117, no. 2, pp. 448-456, 2007.

[78] W. Lin, P. E. Kunkler, H. P. Harding, D. Ron, R. P. Kraig, and B. Popko, "Enhanced integrated stress response promotes myelinating oligodendrocyte survival in response to interferon$\gamma$," American Journal of Pathology, vol. 173, no. 5, pp. 1508-1517, 2008.

[79] W. Lin, Y. Lin, J. Li et al., "Oligodendrocyte-specific activation of PERK signaling protects mice against experimental autoimmune encephalomyelitis," The Journal of Neuroscience, vol. 33, pp. 5980-5991, 2013.

[80] V. Valenzuela, E. Collyer, D. Armentano, G. B. Parsons, F. A. Court, and C. Hetz, "Activation of the unfolded protein response enhances motor recovery after spinal cord injury," Cell Death and Disease, vol. 3, no. 2, article e272, 2012.

[81] M. Schröder and R. J. Kaufman, "The mammalian unfolded protein response," Annual Review of Biochemistry, vol. 74, pp. 739-789, 2005.

[82] S. S. Vembar and J. L. Brodsky, "One step at a time: endoplasmic reticulum-associated degradation," Nature Reviews Molecular Cell Biology, vol. 9, no. 12, pp. 944-957, 2008.

[83] C. T. Kwok, A. G. Morris, J. Frampton et al., "Association studies indicate that protein disulfide isomerase is a risk factor in amyotrophic lateral sclerosis," Free Radical Biology \& Medicine, vol. 18, pp. 81-86, 2013.

[84] C. Appenzeller-Herzog and L. Ellgaard, "The human PDI family: Versatility packed into a single fold," Biochimica et Biophysica Acta, vol. 1783, no. 4, pp. 535-548, 2008. 
[85] C. I. Andreu, U. Woehlbier, M. Torres, and C. Hetz, "Protein disulfide isomerases in neurodegeneration: from disease mechanisms to biomedical applications," FEBS Letters, vol. 586, pp. 2826-2834, 2012.

[86] J. D. Atkin, M. A. Farg, B. J. Turner et al., "Induction of the unfolded protein response in familial amyotrophic lateral sclerosis and association of protein-disulfide isomerase with superoxide dismutase 1," The Journal of Biological Chemistry, vol. 281, no. 40, pp. 30152-30165, 2006.

[87] T. Massignan, F. Casoni, M. Basso et al., "Proteomic analysis of spinal cord of presymptomatic amyotrophic lateral sclerosis G93A SOD1 mouse," Biochemical and Biophysical Research Communications, vol. 353, no. 3, pp. 719-725, 2007.

[88] M. Urushitani, S. A. Ezzi, A. Matsuo, I. Tooyama, and J.-P. Julien, "The endoplasmic reticulum-Golgi pathway is a target for translocation and aggregation of mutant superoxide dismutase linked to ALS," FASEB Journal, vol. 22, no. 7, pp. 2476-2487, 2008.

[89] Y. Honjo, S. Kaneko, H. Ito et al., "Protein disulfide isomeraseimmunopositive inclusions in patients with amyotrophic lateral sclerosis," Amyotrophic Lateral Sclerosis, vol. 12, no. 6, pp. 444450, 2011.

[90] A. K. Walker, M. A. Farg, C. R. Bye, C. A. McLean, M. K. Horne, and J. D. Atkin, "Protein disulphide isomerase protects against protein aggregation and is S-nitrosylated in amyotrophic lateral sclerosis," Brain, vol. 133, no. 1, pp. 105-116, 2010.

[91] R. Mandel, H. J.-P. Ryser, F. Ghani, M. Wu, and D. Peak, "Inhibition of a reductive function of the plasma membrane by bacitracin and antibodies against protein disulfide-isomerase," Proceedings of the National Academy of Sciences of the United States of America, vol. 90, no. 9, pp. 4112-4116, 1993.

[92] M. A. Farg, K. Y. Soo, A. K. Walker et al., "Mutant FUS induces endoplasmic reticulum stress in amyotrophic lateral sclerosis and interacts with protein disulfide-isomerase," Neurobiology of Aging, vol. 33, pp. 2855-2868, 2012.

[93] T. Uehara, T. Nakamura, D. Yao et al., "S-Nitrosylated proteindisulphide isomerase links protein misfolding to neurodegeneration," Nature, vol. 441, no. 7092, pp. 513-517, 2006.

[94] X. Chen, X. Zhang, C. Li et al., "S-nitrosylated protein disulfide isomerase contributes to mutant SOD1 aggregates in amyotrophic lateral sclerosis," Journal of Neurochemistry, vol. 124, pp. 45-58, 2013.

[95] B. G. Hoffstrom, A. Kaplan, R. Letso et al., "Inhibitors of protein disulfide isomerase suppress apoptosis induced by misfolded proteins," Nature Chemical Biology, vol. 6, no. 12, pp. 900-906, 2010.

[96] C. Appenzeller-Herzog, J. Riemer, B. Christensen, E. S. Sørensen, and L. Ellgaard, "A novel disulphide switch mechanism in Erol $\alpha$ balances ER oxidation in human cells," EMBO Journal, vol. 27, no. 22, pp. 2977-2987, 2008.

[97] C. Appenzeller-Herzog, J. Riemer, E. Zito et al., "Disulphide production by Erol $\alpha$-PDI relay is rapid and effectively regulated," EMBO Journal, vol. 29, no. 19, pp. 3318-3329, 2010.

[98] S. Chakravarthi and N. J. Bulleid, "Glutathione is required to regulate the formation of native disulfide bonds within proteins entering the secretory pathway," The Journal of Biological Chemistry, vol. 279, no. 38, pp. 39872-39879, 2004.

[99] C. E. Jessop and N. J. Bulleid, "Glutathione directly reduces an oxidoreductase in the endoplasmic reticulum of mammalian cells," The Journal of Biological Chemistry, vol. 279, no. 53, pp. 55341-55347, 2004.
[100] S. N. Molteni, A. Fassio, M. R. Ciriolo et al., "Glutathione limits Erol-dependent oxidation in the endoplasmic reticulum," The Journal of Biological Chemistry, vol. 279, no. 31, pp. 32667-32673, 2004.

[101] M. G. Pollard, K. J. Travers, and J. S. Weissman, "Erolp: a novel and ubiquitous protein with an essential role in oxidative protein folding in the endoplasmic reticulum," Molecular Cell, vol. 1, no. 2, pp. 171-182, 1998.

[102] A. Higa and E. Chevet, "Redox signaling loops in the unfolded protein response," Cell Signalling, vol. 24, pp. 1548-1555, 2012.

[103] A. J. Dorner, L. C. Wasley, P. Raney, S. Haugejorden, M. Green, and R. J. Kaufman, "The stress response in Chinese hamster ovary cells. Regulation of ERp72 and protein disulfide isomerase expression and secretion," The Journal of Biological Chemistry, vol. 265, no. 35, pp. 22029-22034, 1990.

[104] G. Nardo, S. Pozzi, M. Pignataro et al., "Amyotrophic lateral sclerosis multiprotein biomarkers in peripheral blood mononuclear cells," PLoS ONE, vol. 6, no. 10, Article ID e25545, 2011.

[105] H.-X. Deng, W. Chen, S.-T. Hong et al., "Mutations in UBQLN2 cause dominant X-linked juvenile and adult-onset ALS and ALS/dementia," Nature, vol. 477, no. 7363, pp. 211-215, 2011.

[106] F. Fecto, J. Yan, S. P. Vemula et al., "SQSTM1 mutations in familial and sporadic amyotrophic lateral sclerosis," Archives of Neurology, vol. 68, no. 11, pp. 1440-1446, 2011. 

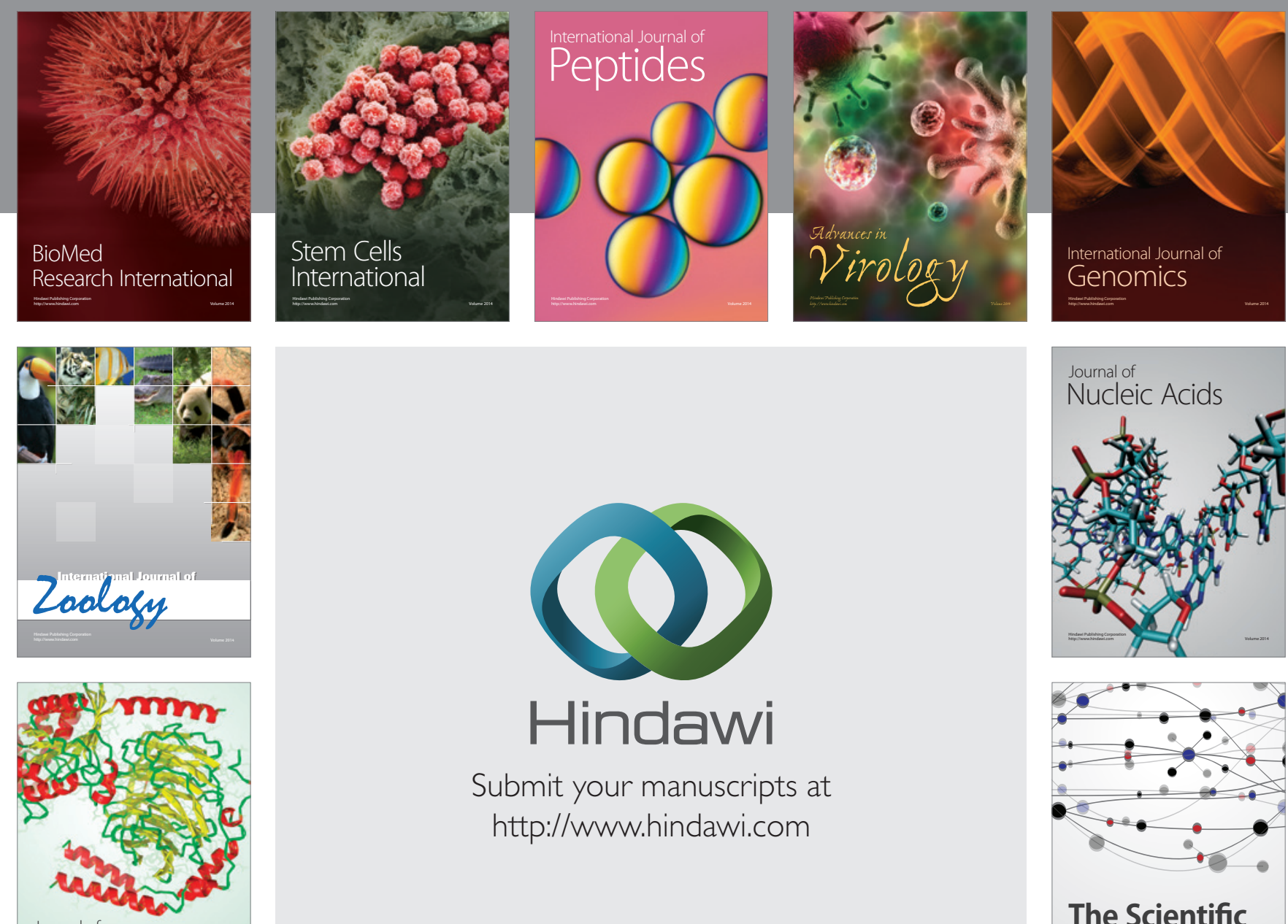

Submit your manuscripts at

http://www.hindawi.com

Journal of
Signal Transduction
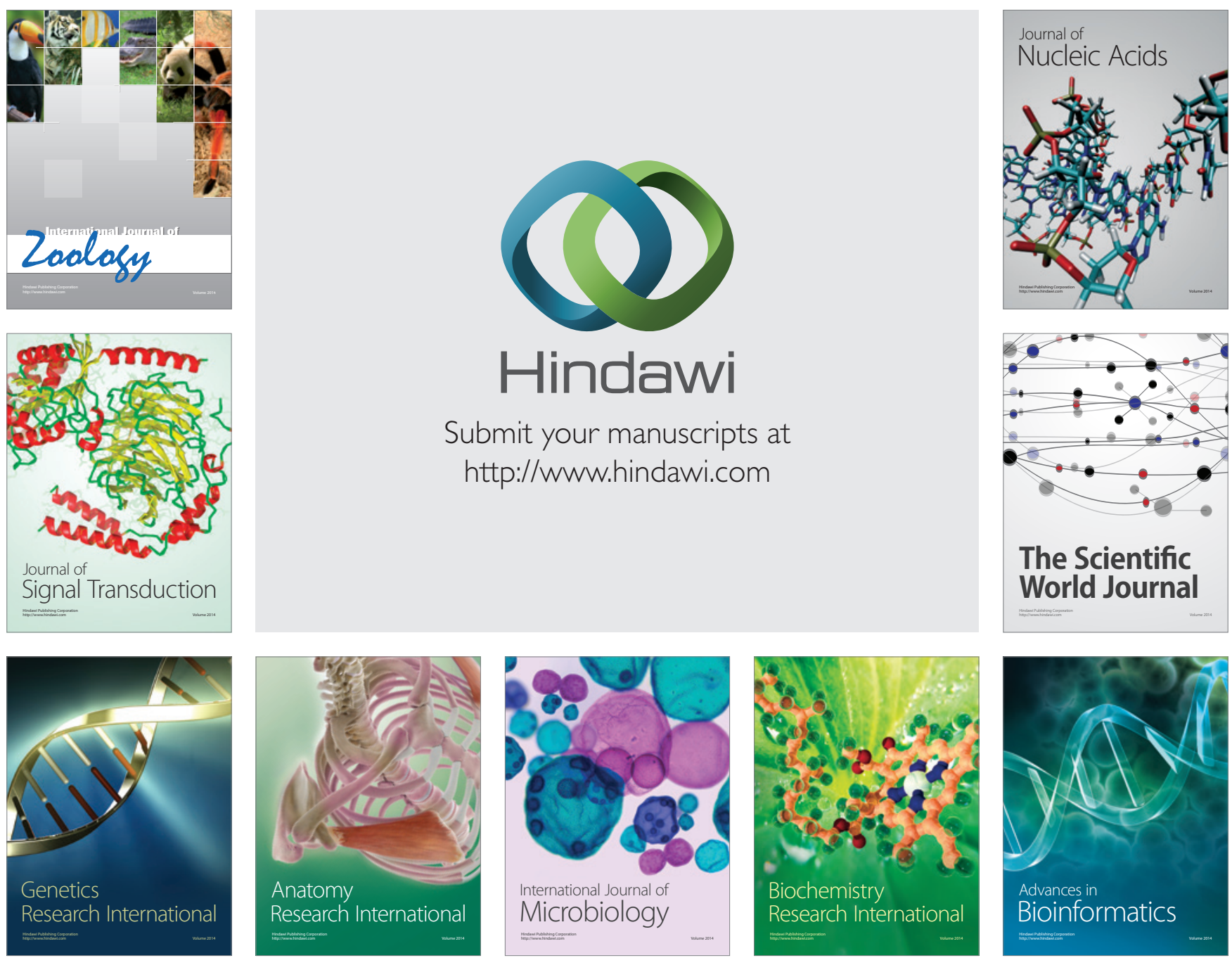

The Scientific World Journal
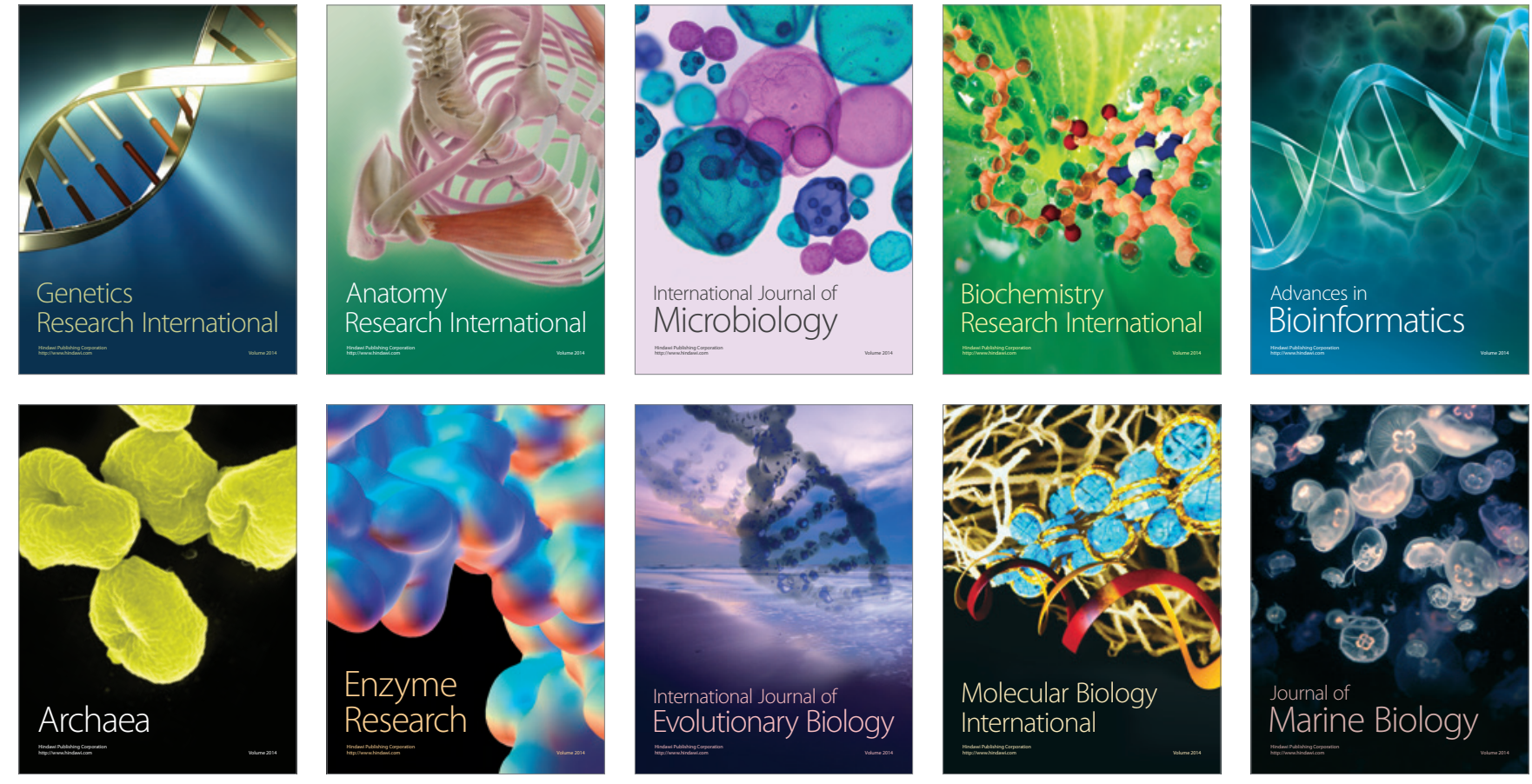